\title{
Estado nutricional de crianças residentes em área de vulnerabilidade social: estudo longitudinal
}

\author{
Nutritional status of children residing in an area of social \\ vulnerability: longitudinal study
}

Dixis Figueroa Pedraza', Ricardo Alves de Olinda1, Thaíse Alves Bezerra1

DOI: 10.1590/0103-1104202012409

RESUMO Objetivou-se analisar a variação dos parâmetros antropométricos, ao longo de dois anos, de crianças menores de cinco anos residentes em uma área de vulnerabilidade, segundo perfil demográfico e de saúde. Realizou-se um estudo longitudinal de 55 famílias com crianças menores de cinco anos e algum membro ex-catador de materiais recicláveis residentes nas proximidades do lixão desativado de Campina Grande, Paraíba. O estado nutricional das crianças foi analisado a partir dos índices Estatura/ Idade (E/I), Peso/Idade (P/I) e Peso/Estatura (P/E), considerando-se as variações dos Escore-Z como variáveis dependentes. Utilizou-se o modelo de efeitos mistos, próprio para avaliar tendência do crescimento em estudo de seguimento. O deficit de estatura prevaleceu nos meninos. A prevalência de baixa estatura na coorte diminuiu de 20,37\% (IC 95\%: 17,40-24,24), em 2012, para 9,26\% (IC 95\%: 7,54-10,58), em 2014, com diferença significativa. Crianças menores de 25 meses tiveram maior variação média de Escore-Z de E/I ( $\mathrm{p}=0,01)$, e os meninos apresentaram maior variação no Escore-Z de E/I ( $\mathrm{p}=0,01)$ e de $\mathrm{P} / \mathrm{E}(\mathrm{p}=0,04)$. Houve diferenças no crescimento das crianças segundo suas características demográficas quando modificadas as condições ambientais insalubres.

PALAVRAS-CHAVE Nível de Saúde. Estado nutricional. Criança. Vulnerabilidade Social. Saneamento.

\begin{abstract}
The aim of this article was to analyze the variation of anthropometric parameters over the course of two years of children under five years of age living in a vulnerability area, according to demographic and health profile. A longitudinal study was conducted involving 55 families with children under five years old and some former garbage digger member living near the deactivated dump of Campina Grande, Paraíba. The nutritional status of the children was analyzed from the Height/Age $(H / A)$, Weight/Age $(W / A)$ and Weight/Height $(W / H)$ indices, considering $Z$-score variations as dependent variables. The mixed effects model was used to evaluate growth tendency in a follow-up study. The diagnosis of height deficit prevailed in male children. The prevalence of stunting in the cohort decreased from $20.37 \%$ (95\% CI: 17,40-24,24), in 2012, to 9.26\% (95\% CI: 7,54-10,58) with significant difference. Children younger than 25 months had higher mean variation in the score- $Z$ of $H / A(p=0,01)$, and boys had higher variation in the score- $Z$ of $H / A(p=$ $0,01)$ and $W / H(p=0,04)$. There were differences in the growth of children living in unhealthy environments according to their demographic characteristics when the environmental conditions were modified.
\end{abstract}

1 Universidade Estadual da KEYWORDS Health Status. Nutritional status. Child. Social Vulnerability. Sanitation. 


\section{Introdução}

O acompanhamento do crescimento físico constitui um dos indicadores mais importantes sobre a saúde da criança. As obtenções de medidas repetidas para esses fins possibilitam melhor consistência na avaliação'.

Tanto o deficit de estatura quanto o deficit de peso são problemas de saúde pública global presentes em todas as regiões do mundo e na maioria dos países e contextos em desenvolvimento, substancialmente mais prevalentes entre crianças pobres do que ricas. Esses problemas relacionam-se ao aumento da morbimortalidade em crianças e a problemas de desenvolvimento ${ }^{2-4}$. Dessa forma, após muitos anos de negligência, o deficit de estatura constitui uma prioridade e meta atual de saúde global ${ }^{5}$.

Os deficits de estatura e peso constituem problemas multicausais que dividem causas em comum, que incluem determinantes ambientais de infecção, inflamação e subnutrição como fatores subjacentes ${ }^{3,6,7}$. Processos infecciosos e desnutrição influenciam-se mutuamente na forma de um círculo vicioso, no qual a infecção diminui o apetite e a absorção intestinal e aumenta o catabolismo ${ }^{6}$. Comparações entre países sugeriram que a defecação aberta pode estar relacionada com o deficit de estatura em grande parcela das crianças ${ }^{8}$. Assim, as condições adequadas de água, saneamento, higiene, eliminação das fezes e remoção de resíduos animais são fatores críticos e medidas de prevenção de extrema importância para o controle da desnutriçãa $0^{3,6,7,9}$.

Outras evidências mostram que as crianças vivendo em favelas, sem condições adequadas de água e saneamento, apresentam maior probabilidade de sofrerem desnutrição ${ }^{10}$. No Brasil, revisão sistemática da literatura, de estudos publicados entre 2006 e 2014, apontou chances maiores de deficit de estatura em amostras obtidas de populações em iniquidade social, de 3,52 (IC95\% 1,35; 10,31), com relação a dados nacionais, e de 2,38 (IC95\% $1,03 ; 6,01)$ tendo como referência os resultados de estudos de base populacional' ${ }^{11}$. Entretanto, a referida pesquisa não considerou amostras procedentes de grupos marginalizados que vivem em favelas.

Em 2012, quase 33\% da população urbana de países em desenvolvimento residiam em favelas. Estima-se que essa população seja de aproximadamente dois bilhões de pessoas em 2030, o que representa meio bilhão de pessoas a mais do que em 2012. Todos os dias, mais de 100 mil pessoas migram para as favelas no mundo em desenvolvimento ${ }^{\mathbf{1 2}, 13}$.

Segundo informações de revisão sistemática da literatura internacional ${ }^{9}$, o objetivo estabelecido pela Assembleia Mundial da Saúde de reduzir, até 2025, o deficit de estatura em crianças menores de cinco anos em $40 \%^{\mathbf{1 4}}$ não seria atingido com os avanços realizados, cujas estimativas indicam redução pequena de aproximadamente $1,8 \%$ ao ano ${ }^{15}$. Assim, o atingimento desse objetivo estaria necessariamente condicionado à combinação de intervenções e programas específicos de nutrição e de outras intervenções relacionadas à nutrição que incorporem ações nutricionais vinculadas aos determinantes subjacentes da desnutrição. As melhorias das condições de água, saneamento e higiene formam parte do leque de intervenções não específicas de nutrição, mas relacionadas a ela, que são necessárias para otimizar a redução das prevalências de deficit de estatura nas crianças menores de cinco anos.

Com relação com essa conjuntura, no Brasil, foi instituída, em 2010, a Política Nacional de Resíduos Sólidos. Tal Política inclui a proteção da saúde pública e da qualidade ambiental entre seus objetivos, constitui um dos marcos mais importantes na história da gestão e do gerenciamento dos resíduos sólidos brasileiros e representa um mecanismo de inclusão social de catadores de materiais recicláveis ${ }^{16}$.

Considerando a importância de estudos longitudinais em desfechos que variam ao longo do tempo, este estudo teve por objetivo analisar a variação dos parâmetros antropométricos, ao longo de dois anos, de crianças menores de cinco anos residentes em uma área de vulnerabilidade segundo o perfil demográfico e de saúde. 


\section{Material e métodos}

\section{Desenho do estudo}

Trata-se de um estudo longitudinal com abordagem quantitativa, desenvolvido nos meses de junho a agosto de 2012 e 2014, com famílias residentes em favela localizada nas proximidades do lixão desativado de Campina Grande, Paraíba. O estudo faz parte do projeto 'Perfil nutricional de crianças menores de cinco anos residentes em área de vulnerabilidade social de Campina Grande, Paraíba e situação de (in) segurança alimentar de suas famílias'. A pergunta inicial do estudo foi se houve mudança no estado nutricional das crianças após a desativação do lixão.

\section{Local, população e amostra de estudo}

O município de Campina Grande é o segundo maior do estado da Paraíba, sua área de abrangência é de $594,18 \mathrm{~km}^{2}$, e sua população, em 2010, era de 385.213 habitantes. No referido ano, seu serviço de Atenção Básica de Saúde apresentava 98 Unidades Básicas de Saúde da Família (UBSF), distribuídas em seis distritos sanitários de saúde.

No estudo, foram incluídas todas as famílias cadastradas nas UBSF I e UBSF II do município de estudo que tinham no seu núcleo familiar algum ex-catador de materiais recicláveis do lixão desativado da cidade e crianças menores de cinco anos, as quais foram identificadas a partir das unidades de saúde que as contemplavam, anteriormente citadas. $\mathrm{O}$ lixão era localizado no bairro Mutirão, na Alça Sudoeste, Rodovia BR 230, a uma distância de 8 $\mathrm{km}$ do centro da cidade, ocupando uma área de 35 hectares. O local foi desativado em janeiro de 2012, em obediência à Lei $\mathrm{n}^{0} 12.305 / 2010$, que instituiu a Política Nacional de Resíduos Sólidos ${ }^{16}$. O esquema de obtenção da amostra inicial de estudo foi anteriormente publicado ${ }^{17}$.

A coleta de dados foi realizada em dois momentos. Participaram do estudo 76 crianças, na primeira etapa (2012), e 54 na segunda (2014). As 22 perdas de seguimento ocorreram devido às mudanças das famílias do local de moradia, o que foi confirmado por visita aos domicílios, estratégia adotada para a obtenção de dados quando as mães não compareceram às UBSF.

As características demográficas, da situação de saúde e socioeconômicas das crianças no início do estudo encontram-se disponíveis, destacando-se a vulnerabilidade social e os processos infecciosos, bem como a associação dos índices antropométricos ao baixo peso ao nascer e à ocorrência de diarreia e vermino$\mathrm{se}^{17}$. No segundo momento do estudo, $87 \%$ das crianças tinham idade $>=25$ meses $<60$ meses. Não houve diferenças entre as características das crianças nos dois momentos do estudo nem na comparação das crianças perdidas com as que finalizaram o estudo, segundo o teste de Qui-quadrado ou Exato de Fischer.

\section{Procedimentos para a coleta de informação}

Foi realizado um treinamento com quatro entrevistadores, que receberam um manual de instruções com informações a respeito dos objetivos da pesquisa, da metodologia adotada no trabalho de campo e do instrumento de coleta de dados. O estudo piloto permitiu efetuar os ajustes necessários ao instrumento de coleta de dados e padronizar a equipe de pesquisa.

Para a coleta de dados, foi aplicado um questionário às mães dos núcleos familiares, contendo informações sobre o sexo (masculino, feminino) e a idade ( $>=25$ meses e $<60$ meses) das crianças. A idade foi calculada considerando as datas da entrevista e de nascimento da criança.

Nas crianças, foram realizadas aferições de peso, comprimento (menores de 24 meses) e estatura (maiores de 24 meses), obedecendo aos procedimentos recomendados pela Organização Mundial da Saúde ${ }^{18}$. Para aferir o peso, foi utilizada balança digital da marca Tanita UM-080 ${ }^{\circledR}$, com variação de 100 gramas. O peso das crianças menores de 24 meses foi obtido através da 
diferença entre a pesagem conjunta da mãe com a criança em seu colo e o valor do peso da mãe. O comprimento foi aferido com auxílio de um infantômetro de madeira portátil com amplitude de $150 \mathrm{~cm}$ e subdivisões de $0,1 \mathrm{~cm}$. A estatura foi aferida utilizando o estadiômetro WCS, com amplitude de $220 \mathrm{~cm}$ e subdivisões de $1 \mathrm{~mm}$ na posição vertical. Todas as medidas foram realizadas duas vezes, utilizando-se o valor da média. Em todos os momentos do estudo, as medidas foram obtidas pela mesma equipe de antropometristas devidamente treinados.

\section{Análise dos dados}

O estado nutricional das crianças foi expresso em Escore-Z dos índices Estatura/Idade (E/I), Peso/Idade (P/I) e Peso/Estatura (P/E), de acordo com o padrão de referência do Multicentre Growth Study ${ }^{19}$. Os cálculos foram realizados com o programa WHO Anthro, v. 3, 2009. O teste Qui-quadrado foi utilizado para analisar as diferenças na proporção de deficit de estatura $\left(\mathrm{E} / \mathrm{I}<-2\right.$ Escore-Z) ${ }^{\mathbf{1 8}}$ entre os momentos final e inicial da coorte, na amostra total e segundo o sexo. Para os outros desvios antropométricos, as análises não foram realizadas considerando as prevalências inexpressivas ao final do estudo.

As variações dos Escore-Z de E/I, P/I e P/E foram consideradas como variáveis dependentes. Utilizou-se o modelo de efeitos mistos, próprio para avaliar tendência do crescimento em estudo de seguimento com medidas repetidas no tempo para o mesmo indivíduo, permitindo estimar variação na medida basal e a taxa de mudança ao longo do tempo ${ }^{20}$.

Dado o pressuposto de normalidade, utilizou-se o teste de Mauchly, inicialmente, para verificar a condição de esfericidade na matriz de variâncias-covariâncias, como garantia de distribuição F exata nas comparações intraindividuais das medidas antropométricas ao longo do tempo. Posteriormente, oito estruturas de matriz de variâncias-covariâncias residual foram avaliadas: $i$. Componente de Variância, ii. Simetria Composta, iii. Autorregressiva de Primeira Ordem, iv.
Toeplitz, v. Simetria Composta Heterogênea, vi. Huynh-Feldt, vii. Autorregressiva de Primeira Ordem Heterogênea, viii. Não Estruturada. A escolha da matriz adequada, com melhor qualidade do ajuste, baseou-se no Critério de Informação de Akaike.

Após a escolha da estrutura de variâncias-covariâncias, procedeu-se à seleção das variáveis com base na diferença entre modelos aninhados, com e sem a variável a testar. A diferença entre os logaritmos de máxima verossimilhança (- 2log ML) dos dois modelos com distribuição de qui-quadrado, comparada com seus graus de liberdade, permitiu testar a significância. Na análise múltipla de efeitos mistos, o ajuste considerou as variáveis com valores de p menores que 0,20 , até o modelo final das associações, com $\mathrm{p}<0,05$.

As análises estatísticas foram realizadas com o auxílio do programa $\mathrm{R}$ ( $\mathrm{R}$ Core Team, 2017). Consideraram-se significativos os valores de $\mathrm{p}<0,05$.

\section{Aspectos éticos}

O projeto foi aprovado pelo Comitê de Ética em Pesquisa da Universidade Estadual da Paraíba (parecer $n^{0}$ 0035.0.133.000-12). A relevância e os objetivos da pesquisa foram devidamente explicados aos participantes, procedimento após o qual foi assinado o Termo de Consentimento Livre e Esclarecido, condição necessária para a participação no estudo.

\section{Resultados}

De acordo com a E/I, percebe-se que o diagnóstico de deficit de estatura prevaleceu nas crianças do sexo masculino. A prevalência de baixa estatura na coorte diminui de $20,37 \%$ (IC 95\%: 17,40-24,24), em 2012, para 9,26\% (IC 95\%: 7,54-10,58), em 2014, com diferença significativa. As prevalências diminuíram de forma significativa em ambos os sexos, com redução de $50 \%$ para o caso dos meninos e de $66,66 \%$ das meninas (gráfico 1). 
Gráfico 1. Prevalências de deficit de estatura (estatura/idade <-2 Escore-Z) de crianças menores de cinco anos residentes em área de vulnerabilidade social. Campina Grande, Paraíba, 2012-2014

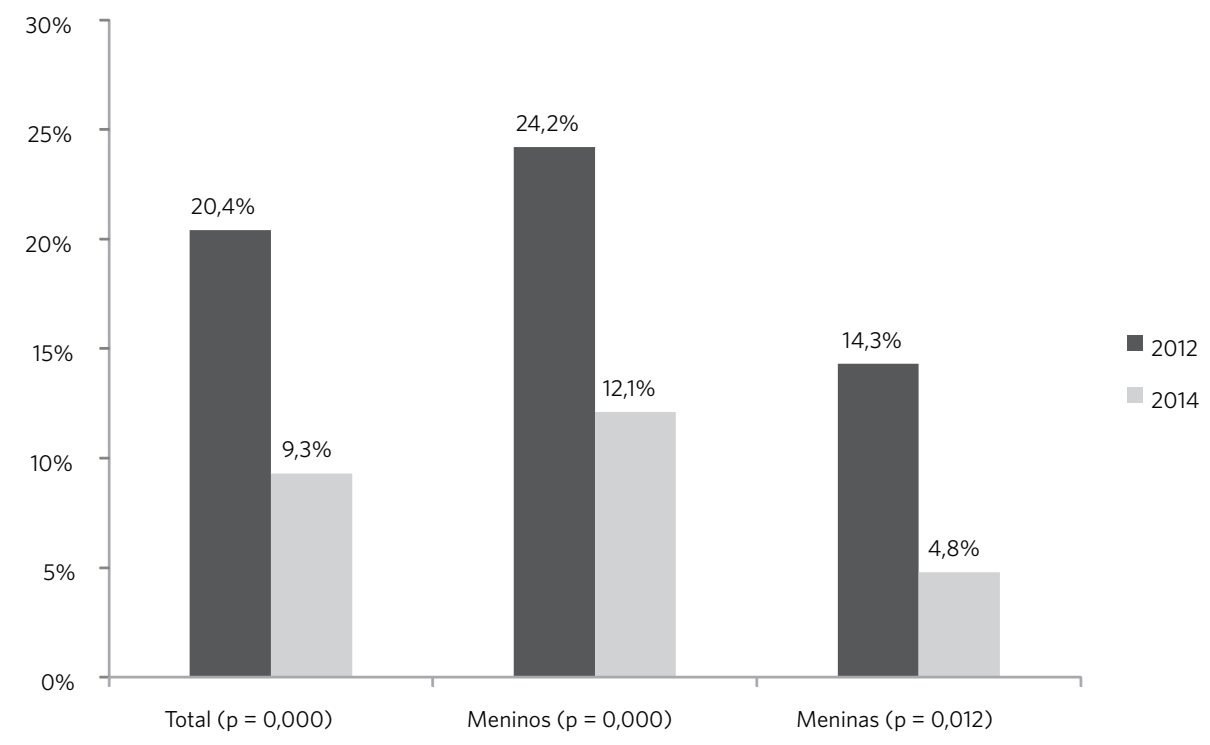

Os resultados da aplicação do teste de esfericidade foram todos significativos $(\mathrm{p}<0,01)$, indicando desvios da estrutura da matriz de variâncias-covariâncias, o que sinaliza a rejeição da condição de esfericidade (tabela 1). O modelo Componente de Variância apresentou o melhor ajuste para a estrutura determinada pelas três medidas dos parâmetros antropométricos do estudo (tabela 2). No teste de significância, verificou-se que apenas o sexo da criança influenciou as variações do seu estado nutricional. Houve efeito significativo $(\mathrm{p}<0,05)$ do sexo da criança nas variações de Escore-Z de E/I e de P/I.

Tabela 1. Teste de esfericidade da estrutura da matriz de variâncias-covariâncias para a variação dos parâmetros antropométricos para medidas repetidas de crianças menores de cinco anos residentes em área de vulnerabilidade social. Campina Grande, Paraíba, 2012-2014

\begin{tabular}{lrrr}
\hline Variáveis & $\mathbf{W}_{1}$ & $\mathbf{X}^{2}$ & p-valor \\
\hline Variação de Estatura/Idade (Escore-Z) & 0,5300 & 32,3759 & $<0,0001$ \\
Variação de Peso/Idade (Escore-Z) & 0,8351 & 9,0095 & $<0,0111$ \\
Variação de Peso/Estatura (Escore-Z) & 0,7839 & 12,1720 & $<0,0023$ \\
\hline
\end{tabular}

$W_{1}$ : Valores da estatística de Mauchly resultantes do ajuste do modelo multivariado para a verificação da condição de esfericidade; $X^{2}$ valores de Qui-quadrado para o teste da razão de verossimilhanças (modelo univariado), com respectivos p-valores resultantes do ajuste dos modelos mistos. 
Tabela 2. Classificação de modelos com diferentes estruturas de matriz de variâncias-covariâncias, obtida nas análises de variação dos parâmetros antropométricos para medidas repetidas de crianças menores de cinco anos residentes em área de vulnerabilidade social. Campina Grande, Paraíba, 2012-2014

\begin{tabular}{lrrrrrrrrr}
\hline Variáveis & Modelos & UN & ARH & SCH & HF & Arpo & Toep & SC & CV \\
\hline Variação de & NP & 6 & 4 & 4 & 4 & 2 & 3 & 2 & 1 \\
Estatura/Idade & -2Reml & 454,1 & 454,1 & 454,1 & 454,1 & 455,2 & 455,2 & 455,2 & 455,2 \\
(Escore-Z) & AIC & 466,1 & 462,1 & 462,1 & 462,1 & 459,2 & 461,2 & 459,2 & 457,2 \\
& NP & 6 & 4 & 4 & 4 & 2 & 3 & 2 & 1 \\
Variação de & -2Reml & 438,8 & 438,8 & 438,8 & 438,8 & 439,4 & 439,4 & 439,4 & 439,4 \\
$\begin{array}{l}\text { Peso/Idade } \\
\text { (Escore-Z) }\end{array}$ & AIC & 450,8 & 446,8 & 446,8 & 446,8 & 443,4 & 445,4 & 443,4 & 441,4 \\
& NP & 6 & 4 & 4 & 4 & 2 & 3 & 2 & 1 \\
$\begin{array}{l}\text { Variação de } \\
\text { Peso/Estatura }\end{array}$ & -2Reml & 422,2 & 422,2 & 422,2 & 422,2 & 424,0 & 424,0 & 424,0 & 424,0 \\
(Escore-Z) & AIC & 434,2 & 430,2 & 430,2 & 430,2 & 428,0 & 430,0 & 428,0 & 426,0 \\
\hline
\end{tabular}

NP: Número de parâmetros do modelo; Reml: Valor que maximiza o logaritmo da função de verossimilhança; AIC: Critério de Informação de Akaike para geração de valores comparáveis e seleção do modelo; UN: Não Estruturada; ARH: Autorregressiva de Primeira Ordem Heterogênea; SCH: Simetria Composta Heterogênea; HF: Huynh-Feldt; Arpo: Auto-regressiva de Primeira Ordem; Toep: Toeplitz; SC: Simetria Composta; CV: Componente de Variância.

Os resultados da análise bivariada indicaram que menor idade da criança e sexo masculino se mostraram inibidores do crescimento linear. Associação similar foi encontrada para o crescimento ponderal com relação ao sexo da criança. Os resultados da análise multivariada confirmaram essas associações: crianças menores de 25 meses tiveram maior variação média de Escore-Z de E/I ( $p$ = 0,01), e os meninos, quando comparados com as meninas, apresentaram maior variação média de Escore- $Z$ de $\mathrm{E} / \mathrm{I}$ ( $\mathrm{p}$ = 0,01) e de $\mathrm{P} / \mathrm{E}(\mathrm{p}=0,04)$ (tabela 3$)$.

Tabela 3. Associação do perfil demográfico e de saúde de crianças menores de cinco anos residentes em área de vulnerabilidade social com a variação dos parâmetros antropométricos ao longo de um ano. Campina Grande, Paraíba, 2012-2014

\begin{tabular}{|c|c|c|c|c|c|c|c|c|c|}
\hline \multirow[t]{3}{*}{ Variáveis } & \multicolumn{9}{|c|}{ Variações médias (Escore-Z) } \\
\hline & \multicolumn{2}{|c|}{ Estatura/Idade (IC 95\%) } & \multirow[t]{2}{*}{ p } & \multicolumn{2}{|c|}{ Peso/Idade (IC 95\%) } & \multirow[t]{2}{*}{$\mathrm{p}$} & \multicolumn{2}{|c|}{ Peso/Estatura (IC 95\%) } & \multirow[t]{2}{*}{$p$} \\
\hline & Não ajustada & Ajustada* & & Não ajustada & Ajustada* & & Não ajustada & Ajustada* & \\
\hline Intercepto & & $\begin{array}{c}-0,21 \\
(-0,52 ;-0,08)\end{array}$ & $<0,001$ & & $\begin{array}{c}0,12 \\
(-0,14 ; 0,24)\end{array}$ & $<0,001$ & & $\begin{array}{c}0,19 \\
(-0,08 ; 0,26)\end{array}$ & $<0,001$ \\
\hline \multicolumn{10}{|l|}{ Idade } \\
\hline \multicolumn{10}{|c|}{$\begin{array}{l}>=25 \text { meses e } \\
<60 \text { meses }^{a}\end{array}$} \\
\hline$<25$ meses & $\begin{array}{c}-0,28 \\
(-0,47 ;-0,09)\end{array}$ & $\begin{array}{c}-0,23 \\
(-0,41 ;-0,06)\end{array}$ & 0,01 & $\begin{array}{c}0,13 \\
(-0,25 ; 0,27) \\
\end{array}$ & $\begin{array}{c}0,17 \\
(-0,22 ; 0,34)\end{array}$ & 0,10 & $\begin{array}{c}0,20 \\
(-0,04 ; 0,44)\end{array}$ & $\begin{array}{c}0,25 \\
(-0,01 ; 0,40)\end{array}$ & 0,07 \\
\hline \multicolumn{10}{|l|}{ Sexo } \\
\hline \multicolumn{10}{|l|}{ Feminino $^{a}$} \\
\hline Masculino & $\begin{array}{c}0,21 \\
(-0,07 ; 0,29)\end{array}$ & $\begin{array}{c}0,24 \\
(-0,08 ; 0,23)\end{array}$ & 0,01 & $\begin{array}{c}0,14 \\
(-0,04 ; 0,22)\end{array}$ & $\begin{array}{c}0,15 \\
(-0,02 ; 0,19)\end{array}$ & 0,33 & $\begin{array}{c}0,25 \\
(-0,06 ; 0,37)\end{array}$ & $\begin{array}{c}0,29 \\
(-0,04 ; 0,31)\end{array}$ & 0,04 \\
\hline
\end{tabular}


Tabela 3. (cont.)

\begin{tabular}{|c|c|c|c|c|c|c|c|c|c|}
\hline \multirow[t]{3}{*}{ Variáveis } & \multicolumn{9}{|c|}{ Variações médias (Escore-Z) } \\
\hline & \multicolumn{2}{|c|}{ Estatura/Idade (IC 95\%) } & \multirow[t]{2}{*}{$\mathrm{p}$} & \multicolumn{2}{|c|}{ Peso/Idade (IC 95\%) } & \multirow[t]{2}{*}{$\mathrm{p}$} & \multicolumn{2}{|c|}{ Peso/Estatura (IC 95\%) } & \multirow[t]{2}{*}{$\mathrm{p}$} \\
\hline & Não ajustada & Ajustada* & & Não ajustada & Ajustada* $^{\star}$ & & Não ajustada & Ajustada* & \\
\hline \multicolumn{10}{|c|}{ Diarreia no último ano } \\
\hline \multicolumn{10}{|l|}{ Nãoa } \\
\hline Sim & $\begin{array}{c}-0,12 \\
(-0,30 ; 0,06)\end{array}$ & $\begin{array}{c}-0,11 \\
(-0,32 ; 0,09)\end{array}$ & 0,26 & $\begin{array}{c}0,18 \\
(-0,18 ; 0,35)\end{array}$ & $\begin{array}{c}0,14 \\
(-0,14 ; 0,28)\end{array}$ & 0,22 & $\begin{array}{c}0,29 \\
(-0,66 ; 1,24)\end{array}$ & $\begin{array}{c}0,26 \\
(-0,56 ; 1,16)\end{array}$ & 0,26 \\
\hline \multicolumn{10}{|c|}{ Catarro/tosse no último ano } \\
\hline \multicolumn{10}{|l|}{ Nãoa } \\
\hline $\operatorname{Sim}$ & $\begin{array}{c}-0,06 \\
(-0,24 ; 0,12)\end{array}$ & $\begin{array}{c}-0,03 \\
(-0,20 ; 0,06)\end{array}$ & 0,39 & $\begin{array}{c}0,06 \\
(-0,27 ; 0,38)\end{array}$ & $\begin{array}{c}0,09 \\
(-0,21 ; 0,30)\end{array}$ & 0,28 & $\begin{array}{c}0,13 \\
(-0,35 ; 0,62)\end{array}$ & $\begin{array}{c}0,14 \\
(-0,32 ; 0,60)\end{array}$ & 0,17 \\
\hline \multicolumn{10}{|c|}{ Verminose no último ano } \\
\hline \multicolumn{10}{|l|}{ Nãoa } \\
\hline $\operatorname{Sim}$ & $\begin{array}{c}-0,05 \\
(-0,20 ; 0,10)\end{array}$ & $\begin{array}{c}-0,09 \\
(-0,15 ; 0,06)\end{array}$ & 0,24 & $\begin{array}{c}0,19 \\
(-0,31 ; 0,36)\end{array}$ & $\begin{array}{c}0,24 \\
(-0,28 ; 0,33)\end{array}$ & 0,41 & $\begin{array}{c}0,22 \\
(-0,37 ; 0,29)\end{array}$ & $\begin{array}{c}0,26 \\
(-0,38 ; 0,25)\end{array}$ & 0,27 \\
\hline
\end{tabular}

a = categoria de referência; *Análise múltipla de efeitos mistos (todas as análises foram ajustadas, também, para as características socioeconômicas das famílias: escolaridade materna, estado civil da mãe, destino domiciliar do lixo, tratamento domiciliar da água para beber e renda domiciliar per capita).

\section{Discussão}

De forma contrária à maioria dos estudos sobre o crescimento das crianças, que se baseiam em informações de delineamentos transversais, com limitações no estabelecimento da direção das associações, este estudo utilizou dados longitudinais que representam mais adequadamente o dinamismo desse processo ${ }^{21}$. Nesse sentido, os resultados do presente estudo mostram, em primeiro lugar, diminuição significativa da prevalência de deficit de estatura tanto na amostra total quanto para ambos os sexos. Apesar de não ser possível afirmar que as modificações podem estar ocorrendo devido à desativação do lixão na zona de residência das crianças do estudo, uma vez que outros fatores não considerados podem exercer influência importante ${ }^{22}$, há de se ressaltar a importância de se considerarem os fatores ambientais e as condições de saneamento, assim como a intensidade e a duração da exposição, como componentes essenciais nos impactos sobre o estado nutricional das crianças $\mathbf{8 , 9 , 2 3 , 2 4}$. As características antropométricas são resultado do ajustamento do potencial genético de crescimento com as condições ambientais ${ }^{25}$.

Segundo os resultados apresentados, diferenças no crescimento linear das crianças estiveram condicionadas ao sexo da criança. Esse achado coincide com os obtidos por outros pesquisadores em estudos anteriores, com desenhos similares $\mathbf{1 , 2 0 , 2 6}$. Estudos de revisão também têm relatado essa associa-

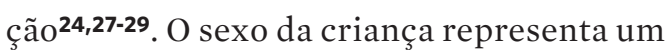
dos fatores de risco com maior influência no crescimento ${ }^{30}$. Sugere-se que crianças do sexo masculino são mais vulneráveis a ambientes desfavoráveis, a condições inadequadas de saneamento e às desigualdades em saúde ${ }^{20,31}$. Por sua vez, meninas caracterizam-se por menor peso ao nascer, o que pode gerar aceleração compensatória ${ }^{1}$. Ainda, os resultados podem estar condicionados à pior situação nutricional dos meninos no início do estudo, visto que crianças com 
deficiências nutricionais mais intensas tendem a apresentar melhores respostas em condições benéficas ao crescimento ${ }^{22}$. Diante do exposto, parece ser esperado observar maiores mudanças de crescimento entre os meninos quando melhoradas as condições do ambiente.

A idade da criança também é considerada uma variável não modificável de grande importância na estatura da criança, sobretudo em situações sociais de vulnerabilidade $\mathbf{2 8 , 3 0}^{\mathbf{3}}$. Esse resultado pode ser decorrente de maior suscetibilidade nas crianças mais novas por consequência de processos como a introdução de alimentos de forma complementar, a maior exposição a enfermidades e a independência que vai sendo adquirida, que repercute numa maior interação com o meio ambiente ${ }^{\mathbf{6}, 28}$. Também deve-se cogitar a relação com o período de mil dias de oportunidades para intervenções nutricionais, onde há uma capacidade de crescimento compensador após restrições no crescimento ${ }^{32}$. Outros estudos, de maneira similar ao presente, têm mostrado o risco do deficit de crescimento associado à idade da criança ${ }^{22-24,28}$.

Com relação ao $\mathrm{P} / \mathrm{E}$ da criança, o presente estudo mostrou, também, associação do Escore-Z com o sexo da criança, com maior variação nos meninos do que nas meninas. Em outro estudo, que analisou a velocidade de crescimento das crianças, os pesquisadores encontraram resultados similares, discutindo sua plausibilidade em função das maiores estruturas corporais e tecido muscular, bem como maior peso ao nascer e rápida velocidade de crescimento nas crianças do sexo masculino ${ }^{1}$. Coerentemente, com foco em países de renda baixa e média, outros autores constataram que os problemas de peso são maiores em meninos do que em meninas ${ }^{27}$.

Embora neste estudo não tenha sido constatada associação entre a diarreia, catarro/tosse e verminose com as variações nos índices antropométricos, sabe-se que os processos infecciosos influenciam de maneira importante o potencial de crescimento $^{\mathbf{6}, 23}$. A infecção na criança produz redução do apetite, diminuição da absorção intestinal e aumento do catabolismo e das necessidades de nutrientes para o crescimento e o sistema imunológico ${ }^{21,28}$. Essa relação é permeada pelas condições de vida, ambientais e de saúde. Melhores condições de saneamento básico (água, lixo e esgoto) constituem uma importante medida para a prevenção de doenças nas crianças menores de cinco anos ${ }^{\mathbf{6} 28}$. Assim, neste estudo, a ausência de associação entre as condições infecciosas e o estado nutricional talvez esteja relacionada à melhoria da situação de saúde das crianças (menores frequências de características de processos infecciosos) ao longo da coorte (resultados complementares do estudo não mostrados).

É oportuno destacar limitações do presente estudo, dada a ausência de análises para alguns parâmetros importantes no crescimento, como o consumo alimentar. Ainda, a impossibilidade de esclarecer as variações do crescimento em termos dos possíveis benefícios gerados por melhorias nas condições do meio ambiente.

\section{Conclusões}

O caráter longitudinal deste estudo permitiu verificar variações no crescimento de crianças residentes em ambientes insalubres, sendo evidenciadas diferenças segundo as características demográficas das crianças quando modificadas as condições ambientais. Maiores variações de peso e comprimento/estatura foram constatadas entre os meninos. Ainda, menor idade da criança representou maior variação no comprimento/estatura. Assim, mudanças no estado nutricional da criança, se modificadas as condições ambientais, podem estar relacionadas à vulnerabilidade implícita nas características demográficas, circunstanciada à situação de saúde da criança. 
Destaca-se a importância deste es-tudo tendo em vista o percentual considerável de famílias vivendo em condições ambientais insalubres no Brasil.

\section{Colaboradores}

Figueroa Pedraza D (0000-0002-5394-828X)* contribuiu para a concepção do estudo; análise e interpretação dos dados; elaboração do rascunho e revisão crítica do conteúdo; e aprovação da versão final do manuscrito. Olinda RA (0000-0002-0509-8428)* contribuiu para a análise dos dados; revisão crítica do conteúdo; e aprovação da versão final do manuscrito. Bezerra TA (0000-0003-3242-4468)* contribuiu para a análise e interpretação dos dados; elaboração do rascunho e revisão crítica do conteúdo; e aprovação da versão final do manuscrito.

\section{Referências}

1. Fonseca PCA, Carvalho CA, Ribeiro SAV, et al. Determinantes da velocidade média de crescimento de crianças até seis meses de vida: um estudo de coorte. Ciênc. Saúde Colet. 2017; 22(8):2713-26.

2. Haddad L, Achadi E, Bendech MA, et al. The Global Nutrition Report 2014: actions and accountability to accelerate the world's progress on nutrition. J Nutr. 2015; 145(4):663-71.

3. Goudet SM, Griffiths PL, Bogin BA, et al. Nutritional interventions for preventing stunting in children (0 to 5 years) living in urban slums. Cochrane Database of Systematic Reviews. 2015 [acesso em 2018 set 22]; 5:CD011695. Disponível em: https://www.ncbi. nlm.nih.gov/pmc/articles/PMC6513167/.

4. Barros FC, Victora CG, Scherpbier R, et al. Socioeconomic inequities in the health and nutrition of children in low/middle income countries. Rev. Saúde Públ. 2010; 44(1):1-16.
5. World Health Organization. Nutrition global targets 2025. Geneva: WHO; 2016. [acesso em 2018 set 22]. Disponível em: http://www.who.int/nutrition/topics/nutrition_globaltargets2025/en/.

6. Stewart CP, Iannotti L, Dewey KG, et al. Contextualising complementary feeding in a broader framework for stunting prevention. Matern Child Nutr. 2013; 9(supl2):27-45.

7. Khara T, Dolan C. Technical Briefing Paper: Associations between Wasting and Stunting, policy, programming and research implications [internet]. 2014. [acesso em 2018 set 22]. Disponível em: https://www. ennonline.net/attachments/1862/WAST_140714.pdf.

8. Spears D. How much international variation in child height can sanitation explain? Policy Research. Working Paper 6351. Washington, DC; World Bank: 2013. and Contributor ID). 
9. Hossain M, Choudhury N, Abdullah KAB, et al. Evidence-based approaches to childhood stunting in low and middle income countries: a systematic review. Arch Dis Child. 2017; 102:903-9.

10. Unger A. Children's health in slum settings. Archives Disease Childhood. 2013; 98(10):799-805.

11. Sousa CPC, Olinda RA, Figueroa Pedraza D. Prevalence of stunting and overweight/obesity among Brazilian children according to different epidemiological scenarios: systematic review and meta-analysis. Sao Paulo Med J. 2016; 134(3):251-62.

12. United Nations. The Millennium Development Goals Report 2012. New York: United Nations; 2012.

13. British Red Cross. Learning from the City: British Red Cross Urban Learning Project Scoping Study. London: British Red Cross; 2012.

14. McGuire S. World Health Organization. Comprehensive Implementation Plan on Maternal, Infant, and Young Child Nutrition. Adv Nutr. 2015; 6:134-5.

15. Onis M, Dewey KG, Borghi E, et al. The World Health Organization's global target for reducing childhood stunting by 2025: rationale and proposed actions. Matern. Child Nutr. 2013; 9(supl2):6-26.

16. Brasil. Lei ${ }^{\circ} 12.305$, de 2 de agosto de 2010. Institui a Política Nacional de Resíduos Sólidos; altera a Lei $\mathrm{n}^{\circ}$ 9.605, de 12 de fevereiro de 1998; e dá outras providências [internet]. Diário Oficial da União. 2 Ago 2010. [acesso em 2020 jan 20]. Disponível em: http:// www.planalto.gov.br/ccivil_03/_ato2007-2010/2010/ lei/l12305.htm.

17. Figueroa Pedraza D, Bezerra TA. Nutritional status of children under five years living in area of social vulnerability of Campina Grande, Paraíba State. Acta Scientiarum. Health Sciences. 2016; 38(1);81-8.

18. World Health Organization. Physical status: The use and interpretation of anthropometry: report of an Expert Committee. Geneva: WHO; 1995.
19. Onis M, Onyango AW, Van den Broeck J, et al. Measurement and standardization protocols for anthropometry used in the construction of a new international growth reference. Food Nutr. Bull. 2004; 25(supl1):15-27.

20. Fausto MA, Carneiro M, Antunes CM, et al. O modelo de regressão linear misto para dados longitudinais: uma aplicação na análise de dados antropométricos desbalanceados. Cad. Saúde Pública. 2008; 24(3):51324.

21. Figueroa Pedraza D. Hospitalização por doenças infecciosas, parasitismo e evolução nutricional de crianças atendidas em creches públicas. Ciênc. Saúde Colet. 2017; 22(12):4105-14.

22. Goulart RMM, França Júnior I, Souza MFM. Fatores associados à recuperação nutricional de crianças em programa de suplementação alimentar. Rev Bras Epidemiol. 2009; 12(2):180-94.

23. Phalkeya RK, Aranda-Jana C, Marx S, et al. Systematic review of current efforts to quantify the impacts of climate change on undernutrition. Proc Natl Acad Sci USA. 2015; 112(33):E4522-9.

24. Keino S, Plasqui G, Ettyang G, et al. Determinants of stunting and overweight among young children and adolescents in sub-Saharan Africa. Food Nut. Bull. 2014; 35(2):167-78.

25. Figueroa Pedraza D, Souza MM, Rocha ACD. Fatores associados ao estado nutricional de crianças pré-escolares brasileiras assistidas em creches públicas: uma revisão sistemática. Rev. Nutr., 2015; 28(4):45164.

26. Grace K, Davenport F, Funk C, et al. Child malnutrition and climate in Sub-Saharan Africa: An analysis of recent trends in Kenya. Appl Geogr. 2012; 35(12):405-13.

27. Black RE, Victora CG, Walker SP, et al. Maternal and child undernutrition and overweight in low-income and middle-income countries. The Lancet. 2013; 382:427-51. 
28. Akombi BJ, Agho KE, Hall JJ, et al. Stunting, Wasting and Underweight in Sub-Saharan Africa: A Systematic Review. Int J Environ Res Public Health. 2017; 14:863.

29. Wamani H AA, Peterson S, Tumwine JK, et al. Boys are more stunted than girls in Sub-Saharan Africa: a meta-analysis of 16 demographic and health surveys. BMC Pediatr. 2007; 7:17.

30. Fenske N, Burns J, Hothorn T, et al. Understanding child stunting in India: a comprehensive analysis of socioeconomic, nutritional and environmental determinants using additive quantile regression. PloS One. 2013; 8(11):e78692.
31. Skoufias E, Vinha K. Climate variability and child height in rural Mexico. Econ Hum Biol. 2012; 10(1):5473.

32. Victora C. Los mil días de oportunidad para intervenciones nutricionales. De la concepción a los dos años de vida. Arch Argent Pediatr. 2012; 110(4):311-7.

Recebido em 15/03/2019

Aprovado em 04/12/2019

Conflito de interesses: inexistente

Suporte financeiro: não houve 